\title{
Peroral endoscopic myotomy (POEM): a comparative study between Chagasic and idiopathic achalasia
}

\section{다 (1) $\odot$}

Authors

Galileu F.A. Farias ${ }^{1}$, Diogo T.H. de Moura',2, Eduardo T.H. de Moura ${ }^{1}$, Daniel T. de Rezende ${ }^{1}$, Kelly E. Hathorn², Ary Nasi $^{1}$, Natália S.F. Queiroz ${ }^{1}$, Eduardo G.H. de Moura ${ }^{1}$

Institutions

1 Gastrointestinal Endoscopy Unit, Hospital das Clínicas da Faculdade de Medicina da Universidade de São Paulo, São Paulo, Brazil

2 Division of Gastroenterology, Hepatology and Endoscopy, Brigham and Women's Hospital, Harvard Medical School, Boston, Massachusetts, United States

submitted 11.7.2019

accepted after revision 24.9.2019

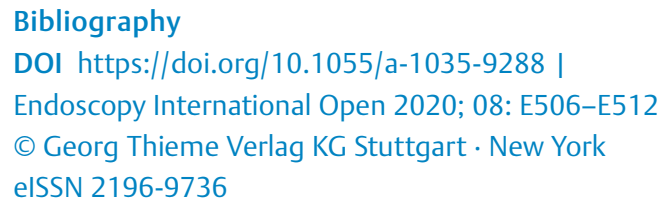

Corresponding author

Galileu F. A. Farias, MD, University of São Paulo Medical School, Gastrointestinal Endoscopy Unit, Dr. Arnaldo Av, 455, São Paulo, 01246-903, Brazil

Fax: +30617000

galileufaf@gmail.com

\section{ABSTRACT}

Background and study aims Achalasia can be classified as either primary (idiopathic) achalasia or secondary achalasia, which is a consequence of another systemic disease.
Peroral endoscopic myotomy (POEM) is an effective and safe treatment for achalasia. We evaluated the efficacy and safety of POEM in patients with Chagasic achalasia compared to idiopathic achalasia.

Patients and methods We evaluated POEM procedures performed at a single institution from November 2016 to January 2018. Demographic data, Eckardt score, lower esophageal sphincter (LES) pressure, body mass index, post-operative erosive esophagitis, adverse events, length of hospital stay, and procedure-related parameters were analyzed.

Results Fifty-one patients underwent POEM as a treatment for achalasia in this period (20 patients with Chagasic and 31 with Idiopathic etiology). The overall clinical success rate was $92.1 \%$, with no statistical difference between groups ( $90 \%$ in the Chagasic group vs. $93.5 \%$ in the Idiopathic group, $P=0.640$ ). Both groups had significant reduction in Eckardt score and in LES pressure, and increase in bodey mass index (BMI) at 1-year follow-up. There was no statistical difference between groups regarding Eckardt score $(P=0.439)$, LES pressure $(p=0.507)$, BMI $(P=0.254)$, post erosive esophagitis ( $35 \%$ vs. $38.7 \%, P=0.789$ ), adverse events ( $30 \%$ vs. $12 \%, P=0.163$,) length of hospital stay (3.75 days vs. 3.58 days, $P=0.622$ ), and operative time (101.3 min vs. $99.1 \mathrm{~min}, P=0.840)$.

Conclusion POEM is an effective and safe treatment for patients with achalasia. There is no difference in POEM outcomes for those patients with Chagasic or Idiopathic achalasia.

\section{Introduction}

Achalasia is a benign esophageal motor disorder, characterized by failure of relaxation of the lower esophageal sphincter (LES) in response to swallowing, which results from degeneration of the neurons of the esophageal wall [1 - 3]. Epidemiological data demonstrate an incidence of approximately 1 in 200,000 people in the United States and Europe, without any predilection for sex or age [3].

Achalasia can be classified as either primary (idiopathic) achalasia, in which the underlying etiology has yet to be clearly defined, or secondary achalasia, which is a consequence of another systemic disease. Chagas disease is the main cause of secondary achalasia, occurring predominantly in Central and South America. Esophageal infection by the protozoan Trypanosoma cruzi can result in loss of intramural ganglion cells, leading to aperistalysis and incomplete relaxation of the LES [4]. Achalasia may cause dysphagia, weight loss, food regurgitation and retrosternal chest pain [5]. Eckardt score is the most commonly used tools to evaluate these symptoms [6,7]. High-resolution esophageal manometry is considered the gold standard method for the diagnosis of achalasia [8]. 
- Table 1 Rezende's classification based on findings on barium esophagogram [12].

Grade I The esophagus shows difficulty emptying and mild hypotonia, with episodes of tertiary waves and no dilation.

Grade II Contraction of the lower esophageal sphincter (achalasia). The esophagus shows a mild to moderate increase in caliber; tertiary waves are more frequent.

Grade III The esophageal lumen shown an evident increase in diameter. The distal portion has the classic "bird beak" sign. The majority of cases with total akinesis of the esophagus show violent contractions of the circular musculature.

Grade IV In addition to the changes described for grade III involvement, we observed intense dilation of the esophagus, which seems to rest on the right phrenic hemidiaphragm. We refer to this as severe (sigmoid) megaesophagus.

Esophagogastroduodenoscopy (EGD) and the barium esophagogram are auxiliary tests. Endoscopic, pharmacological or surgical options are available for treatment of achalasia $[9,10]$. Currently, laparoscopic Heller myotomy (LHM) is considered the standard surgical method. However, in recent years, peroral endoscopic myotomy (POEM) has gained popularity due to its excellent safety and efficacy profile $[8,9]$.

There is only one study [11] to date in the literature which compares the outcomes of POEM in patients treated for Chagasic achalasia versus those treated for Idiopathic achalasia. Thus, we conducted a comparative study to evaluate the efficacy and safety of POEM among patients with Chagasic and Idiopathic achalasia whom were followed for at least 1 year after the POEM procedure.

\section{Patients and methods}

This is a retrospective review of our prospectively collected database of POEM

procedures in patients with achalasia performed at a tertiary-referral center from November 2016 to January 2018. All patients with achalasia, diagnosed based on clinical symptoms, barium esophagogram and conventional esophageal manometry, were included in this study, regardless of whether they had undergone previous treatments for achalasia. All patients with achalasia and serology testing positive for Chagas were considered to have Chagasic achalasia. No patients had been previously included in other published studies. All patients were classified into the stages of Chagas disease based on the findings on barium esophagogram noting the degree of megaesophagus and severity of motor abnormalities according to Rezende's classification [12] ( $\$$ Table 1 ).

Informed consent was obtained before the procedure, after explaining the risks and benefits and the alternative treatment options. This study was approved by ethics committee of the Faculty of Medicine of the University of São Paulo.

The primary outcome was clinical success, defined as Eckardt score $<3$ after treatment at 1 -year follow-up. Eckardt score
- Table 2 Eckardt Score [13].

\begin{tabular}{|l|l|l|l|l|}
\hline Symptom & \multicolumn{3}{|c|}{ Score } & \multicolumn{2}{|l|}{} \\
\hline Dysphagia & $\mathbf{0}$ & $\mathbf{1}$ & $\mathbf{2}$ & $\mathbf{3}$ \\
\hline Regurgitation & None & Occasional & Daily & $\begin{array}{l}\text { With every } \\
\text { meal }\end{array}$ \\
\hline Chest pain & None & Occasional & Daily & $\begin{array}{l}\text { Several times } \\
\text { a day }\end{array}$ \\
\hline $\begin{array}{l}\text { Weight loss } \\
\text { (kg) }\end{array}$ & 0 & $<5$ & $5-10$ & $>10$ \\
\hline
\end{tabular}

consists of 4 components including dysphagia, chest pain, regurgitation, and weight loss. Each component is assigned a score from 0 to 3 based on the patient's self-reported response, resulting in a total score that can vary from 0 to 12 ( $>$ Table 2 ) [13].

Secondary outcomes were LES pressure (analyzed pre-POEM and 1-year post-POEM by conventional manometry), body mass index (BMI), postoperative erosive esophagitis (evaluated with endoscopy and classified according to the Los Angeles classification [14]), adverse events (AEs), length of hospital stay (from the day of procedure until hospital discharge and any readmission related to an adverse event) and procedure-related parameters (including myotomy length (centimeters, cm), orientation of myotomy (anterior or posterior), length of mucosal incision $(\mathrm{cm})$, number of clips to close the incision, operative time (minutes, $\mathrm{min}$ ) and pneumoperitoneum requiring needle decompression). AEs were defined as any symptomatic event requiring temporary stop of the procedure and/or further intervention; any event that prevented completion and/or resulted in prolongation of hospital stay, required another procedure, or subsequent medical consultation. Adverse events were classified according to the ASGE lexicon severity grading system [15]. Pneumoperitoneum requiring needle decompression was not considered an $\mathrm{AE}$ as this is an expected consequence of our procedure.

\section{Procedure}

All patients were started on a liquid diet 48 to 72 hours prior to the procedure and were "nil per os" (NPO) for the 12-hour period prior to the procedure. All patients completed a 5-day course of oral nystatin as prophylaxis for candidiasis prior to the procedure. The endoscope used was a GIF-180H model (Olympus, Tokyo, Japan) and the insufflation was through a $\mathrm{CO}_{2}$ insufflator (OLYMPUS UCR; Olympus, Tokyo, Japan) with an estimated constant flow of 1.2 liters $/ \mathrm{min}$.

All POEM procedures were long $(>7 \mathrm{~cm})$ posterior full-thickness myotomies (FTM) performed by one senior endoscopist. Physiologic saline solution $\mathrm{NaCl} 0.9 \%$ diluted in Indigo Carmine $0.8 \%$ was used to elevate and expose the submucosal space, initially $10 \mathrm{~cm}$ proximal to the LES, followed by a $2-\mathrm{cm}$ longitudinal incision of the mucosa. With the aid of a transparent cap positioned at the end of the device, the endoscope was inserted 


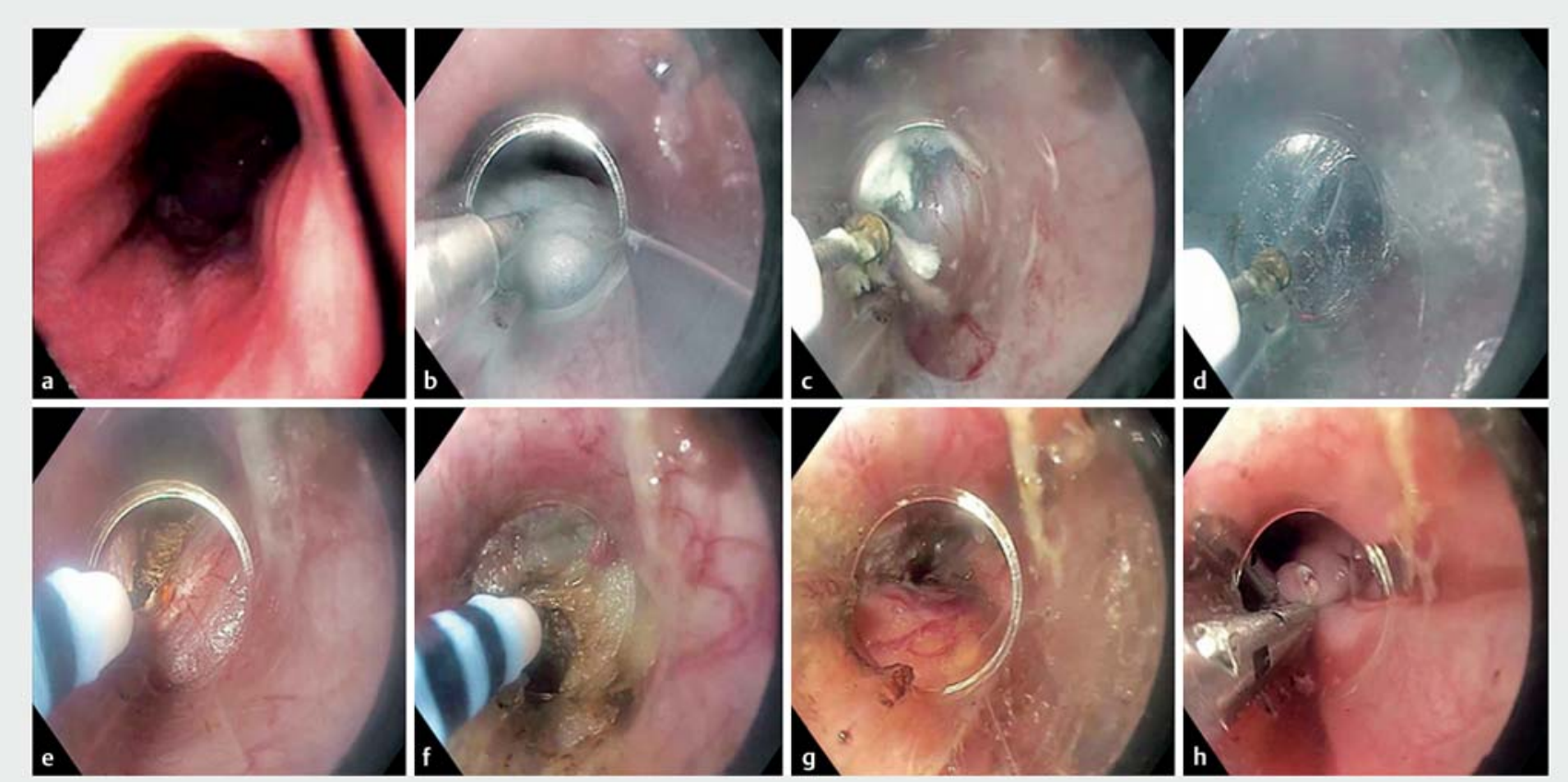

- Fig. 1 POEM procedure. a Endoscopy showed a dilated esophagus. b Submucosal injection in the posterior wall. c Mucosal incision at 6 o'clock position. $\mathbf{d}$ Submucosal dissection. e Section of the circular muscle fibers. $\mathbf{f}$ Section of the longitudinal muscle fibers. $\mathbf{g}$ Aspect after full-thickness myotomy. $\mathbf{h}$ Closure of mucosal incision.

into the space and sequential injection and dissection of the submucosa with Erbe JET (Erbe, Tuebingen, Germany) was performed using coagulation spray (effect 2 and 60 watts), creating an extensive submucosal tunnel along the posterior wall of the esophagus, extending up to 3 to $4 \mathrm{~cm}$ into the cardia, below the LES. In the next step, with the endoscope positioned $2 \mathrm{~cm}$ distal from the incision site of the mucosa, the myotomy (EndoCut Q - effect 2, cutting duration 3 and cutting interval 5) begins with a total section of the circular and longitudinal muscle fibers, extending distally 3 to $4 \mathrm{~cm}$ below the cardia. The length of gastric myotomy was confirmed by markings on the endoscope. An endoscopic evaluation was performed after completion of the submucosal tunnel to identify any evidence of ischemia or mucosal perforation. The access site to the submucosal tunnel was closed with the use of Hemoclips in the distal to proximal direction. At the end of the procedure, the endoscope was again inserted into the natural lumen of the esophagus and used to confirm smooth passage into the stomach ( $\triangleright$ Fig. 1 ).

\section{Postoperative care}

On postoperative day 1 , all patients underwent a barium esophagogram examination. If no evidence of fistula or leak, the patient's diet was advanced to a clear liquid diet, with progression to a full liquid diet at lunch if tolerated. If tolerating a diet and no other procedural-related adverse events, patients could be discharged on postoperative Day 1. The full liquid diet was continued for a total of 14-days post-POEM and progressed then to soft foods and to solids as tolerated. All patients were discharged on proton pump inhibitor (PPI) $40 \mathrm{mg}$ daily for 1 month. If patients had symptoms consistent with gastroesoph- ageal reflux disease (GERD), upper endoscopy was performed, and if erosive esophagitis was demonstrated, PPI was continued for an additional one month.

\section{Follow-up}

Conventional manometry and an esophagogastroduodenoscopy (EGD) were performed in all patients prior to POEM, and in all patients 12 months post-procedure, to evaluate for LES pressure and presence or absence of erosive esophagitis. Patients presenting with GERD symptoms prior to the 1-year follow-up point were referred immediately for EGD. Additionally, Eckart score and BMI were also evaluated at 12 months after POEM.

\section{Statistical analysis}

For quantitative and Gaussian distribution variables, we used Mean and Standard Deviation. For quantitative variables without Gaussian distribution, we use Median and Interquartile Range (the median, instead of the mean, and the range to express the variation that is provided by the standard deviation). For qualitative variables, we used Absolute Frequencies (n) and Percentages. The nonparametric Mann-Whitney test is the Wilcoxon rank-sum test and was used to compare the two groups during the pre- and post-procedure moments. The non-parametric Wilcoxon test is the signed-rank Wilcoxon test and was used to compare the pre- and post- moments in each of the two groups $[16,17]$. SPSS 17.0 software for windows was used for the calculations. The level of significance used for the tests was $<0.05$. 
- Table 3 Patient characteristics.

\begin{tabular}{|c|c|c|c|}
\hline Patient characteristics & Chagas $(n=20)$ & Idiopathic $(n=31)$ & $P$ value \\
\hline Female; n (\%) & $11(55.0)$ & $16(51.6)$ & 0.813 \\
\hline Age; mean + SD & $53.70+11.74$ & $44.61+14.80$ & 0.128 \\
\hline \multicolumn{3}{|l|}{ Rezende grade } & \multirow[t]{5}{*}{0.604} \\
\hline - I; n (\%) & $5(25)$ & $6(19.4)$ & \\
\hline - II; n (\%) & $11(55)$ & $21(67.7)$ & \\
\hline - III; n (\%) & $4(20)$ & $4(12.9)$ & \\
\hline - IV; n (\%) & $0(0.0)$ & $0(0.0)$ & \\
\hline Previous treatment; n (\%) & $2(10)$ & $5(16.1)$ & 0.690 \\
\hline \multicolumn{4}{|l|}{ Pre-POEM } \\
\hline - Eckardt; mean (range) & $7.5(6.0-9.0)$ & $8.0(6.0-9.0)$ & 0.681 \\
\hline - LES pressure; mean+SD & $21.67+13.96$ & $25.21+13.14$ & 0.547 \\
\hline - BMI mean +SD & $25.41+4.60$ & $22.17+5.67$ & 0.254 \\
\hline
\end{tabular}

\section{Results}

\section{Patient characteristics}

Fifty-one patients (52.9\% female, mean age $50 \pm 13.8$ ) underwent POEM as a treatment for achalasia in this period. Twenty patients had a diagnosis of Chagasic achalasia and 31 patients had a diagnosis of Idiopathic achalasia. All procedures were long $(>7 \mathrm{~cm})$ posterior full-thickness POEM. There was no difference between the two groups concerning the degree of the Achalasia (Rezende's classification). Seven (13\%) patients underwent some previous treatment for achalasia. In the Chagasic group, 1 (5\%) patient underwent prior pneumatic dilation and 1 (5\%) patient underwent prior LHM, and in the idiopathic group, 2 (6\%) patients underwent prior pneumatic dilation and $3(10 \%)$ patients underwent prior LHM.

Before the procedure, the mean Eckardt score, LES pressure (LESP) and BMI were 7.5 (6.0-9.0), $21.67(+13.96)$ and 25.21 $(+13.14)$ in the Chagasic group and $8.0(6.0-9.0), 25.41$ $(+4.60)$ and $22.17(+5.67)$ in the Idiopathic group, respectively. Patient characteristics were not significantly different between both groups ( $>$ Table 3 ).

\section{Procedure-related parameters}

A posterior myotomy was performed in all patients. Total myotomy length was $11.75 \mathrm{~cm}(+1.41)$ in the Chagasic group and $11.35 \mathrm{~cm}(+1.25)$ in the idiopathic group. There were no significant differences between groups concerning myotomy length $(P=0.355)$. When dichotomized into esophageal and gastric myotomy lengths specifically, there were no statistically significant differences regarding length of esophageal myotomy $(8.35+1.57$ vs. $7.71+1.22 \mathrm{~cm}$ in the Chagasic vs. Idiopathic group, respectively, $P=0.117)$ or gastric myotomy $(3.40+$ $0.82 \mathrm{~cm}$ vs. $3.71+0.74 \mathrm{~cm}$ in the Chagasic vs. Idiopathic group, respectively, $P=0.171$ ). The length of mucosal incision and number of clips needed to close the incision was $2.40 \mathrm{~cm}$ $(+0.60)$ and 5.35 clips $(+1.53)$ in the Chagasic group and $2.26 \mathrm{~cm}(+0.51)$ and 5.32 clips $(+2.10)$ in the Idiopathic group. There were no significant differences between groups concerning the length of mucosal incision $(P=0.340)$ and number of clips needed to close the incision $(P=0.772)$. Mean operative time for all procedures was $100.07 \mathrm{~min}(+35.18)$; in the Chagasic group, mean operative time was $101.39 \mathrm{~min}(+38.78)$ and in the Idiopathic group the mean operative time was $99.19 \mathrm{~min}$ $(+33.30)$. There was no statistically significant difference between groups concerning operative time $(P=0.840)$. Pneumoperitoneum requiring needle decompression occurred in $18 \mathrm{pa}$ tients (35\%); 7 (35\%) in the Chagasic group and 11 (35.4\%) in the Idiopathic group. There were no significant differences between groups concerning the rates of pneumoperitoneum requiring needle decompression $(P=0.81)$. Procedure-related parameters are summarized in $>$ Table 4.

\section{Clinical success}

POEM was technically successful in all patients (100\%). Clinical success was achieved in 47 of 51 patients (92.1\%). Clinical success was present in 18 of 20 patients (90\%) in the Chagasic group and 29 of 31 patients (93.5\%) in the idiopathic group. There was no statistical difference between groups regarding clinical success $(P=0.640)$.

Both groups had statistically significant reduction in Eckardt score compared to baseline (7.50 vs. 1.00 in the Chagasic group, $P<0.001 ; 8.00$ vs 0.00 in the Idiopathic group, $P<$ $0.001)$. There was no statistical difference between groups regarding post-POEM Eckardt score $(P=0.439)$ at 12 months. 
- Table 4 Procedure-related parameters.

\begin{tabular}{|l|c|c|c|}
\hline Parameter & Chagas $(\mathbf{n = 2 0})$ & Idiopathic $(\mathbf{n = 3 1 )}$ & $\mathbf{P}$ value \\
\hline Length of esophageal myotomy; mean \pm SD $(\mathbf{c m})$ & $8.35 \pm 1.57$ & $7.71 \pm 1.22$ & 0.117 \\
\hline Length of gastric myotomy; mean \pm SD $(\mathrm{cm})$ & $3.40 \pm 0.82$ & $3.71 \pm 0.74$ & 0.171 \\
\hline Length of myotomy; mean \pm SD $(\mathrm{cm})$ & $11.75 \pm 1.41$ & $11.35 \pm 1.25$ & 0.355 \\
\hline Length of mucosal incision; mean \pm SD $(\mathrm{cm})$ & $2.40 \pm 0.60$ & $2.26 \pm 0.51$ & 0.340 \\
\hline Number of clips needed to close the incision; mean \pm SD & $5.35 \pm 1.53$ & $5.32 \pm 2.10$ & 0.772 \\
\hline Operative time; mean \pm SD (min) & $101.39 \pm 38.78$ & $99.19 \pm 33.30$ & 0.840 \\
\hline Pneumoperitoneum requiring needle decompression, $n(\%)$ & $7(35)$ & $11(35.4)$ & 0.812 \\
\hline SD, standard deviation & & & \\
\hline
\end{tabular}

\section{LESP}

Both groups had significant reduction in LESP (21.76 vs. 10.32 in the Chagasic group, $P<0.001 ; 25.21$ vs. 10.70 in the idiopathic group, $P<0.001)$. There was no statistical difference between groups regarding $\operatorname{LESP}(P=0.507)$ at 12 months.

\section{BMI}

Both groups had significant increases in BMI (25.41 vs. 27.11 in the Chagasic group, $P<0.001 ; 22.17$ vs. 25.99 in the idiopathic group, $P<0.001)$ at 12 months. There were no significant differences between groups concerning the BMI $(P=0.254)$.

\section{Length of hospital stay}

Mean length of hospital stay was 3.75 days $(+1.45)$ in the Chagasic group and 3.58 days $(+0.99)$ days in the idiopathic group $(P=0.622)$. There were no significant differences between groups concerning length of hospital stay $(P=0.622)$.

\section{Postoperative erosive esophagitis}

Postoperative esophagitis occurred in 19 patients (37\%); seven of these patients were in the Chagasic group (35\%) and 12 in the idiopathic group (38.7\%) at 12 months. Within the Chagasic group, 5 (25\%) patients had Grade A, 1 (5\%) had Grade C and $1(5 \%)$ had Grade D esophagitis. Within the Idiopathic group, 5 (16\%) patients had Grade A, 2 (6\%) had Grade B, 3 (9\%) had Grade C and 2 (6\%) had Grade D esophagitis. There were no significant differences between groups concerning the rates of erosive esophagitis $(P=0.891)$. Rates of mild $/ \mathrm{mod}$ erate (LA grade A/B) was $25 \%(5 / 20)$ and $22 \%(7 / 31)$ and the rates of severe (LA grade C/D) esophagitis was $10 \%(2 / 20)$ and $16 \%(5 / 31)$ in the Chagasic group and in the idiopathic group, respectively. There were no significant differences between groups concerning the esophagitis degrees (mild/moderate and severe) post-POEM.

\section{Adverse events}

AEs occurred in 10 patients (19\%); six in the Chagasic group (30\%) and four (12\%) in the idiopathic group. In the Chagasic group, $5(25 \%)$ patients had mucosal injury ( 3 gastric and 2 esophageal), and 1 (5\%) patient experienced pulmonary thromboembolism (PE). In the Idiopathic group, 4 (12\%) patients had mucosal injury ( 2 gastric and 2 esophageal.) All mucosal injuries were successfully treated with Hemoclip placement. The patient who experienced PE was treated with therapeutic anticoagulant and was discharged without further complication. There were no significant differences between groups concerning the rates of AEs $(P=0.163)$.

The treatment results are summarized in $>$ Table 5 .

\section{Discussion}

Idiopathic achalasia is the most common type of achalasia, however, the cause for the initial reduction of inhibitory neurons in idiopathic achalasia remains unknown [18]. Chagas disease can cause destruction of the neurons of the enteric nervous system, leading to denervation in both the myenteric and submucosal plexuses. Histologically, neuronal reduction and focal areas of fibrosis in the smooth muscle and myenteric plexuses have been described [19].

Management of patients with Chagasic and idiopathic achalasia is similar, with a primary aim to decrease pressure of the LES for relief of symptoms. No treatment can reverse degeneration of ganglion cells or restore lost esophageal neurons [20, 21].

Traditional treatment methods for achalasia include botulinum toxin injection, pneumatic balloon dilation, and Heller myotomy [22]. However, with advancement in endoscopic procedures, the less-invasive POEM approach has come to the forefront in management of achalasia. In 2010, Inoue et al. [8] performed the world's first POEM in a human for treatment of achalasia. There is only one study to date in the literature which compares the outcomes of POEM in patients treated for Chagasic achalasia versus those treated for Idiopathic achalasia [11].

In our study, we demonstrated a clinical success rate of $90 \%$ $(18 / 20)$ and $93.5 \%(29 / 31)$ in the Chagasic and idiopathic groups, respectively, at 12 -month follow-up. In a systematic review and meta-analysis, Barbieri et al. [23] analyzed 16 studies, including 551 POEM procedures, showing technical and clinical success of $97 \%$ ( $94 \%$ to $98 \%$ ) and $93 \%$ (90\% to 95\%). Thus, our results are similar to those seen in prior studies. Seven of our patients underwent some previous treatment for achalasia. In 
- Table 5 Treatment results.

\begin{tabular}{|c|c|c|c|}
\hline Parameter & Chagas $(n=20)$ & Idiopathic $(n=31)$ & $P$ value \\
\hline Clinical success, n (\%) & $18(90.0)$ & $29(93.5)$ & 0.640 \\
\hline \multicolumn{4}{|l|}{ Post POEM } \\
\hline - Eckardt; mean (range) & $1.0(0.0-1.75)$ & $0.0(0.0-2.0)$ & 0.439 \\
\hline " LES pressure; mean \pm SD & $10.32 \pm 8.13$ & $10.70 \pm 5.33$ & 0.507 \\
\hline - BMI; mean \pm SD & $27.11 \pm 4.62$ & $25.99 \pm 6.18$ & 0.254 \\
\hline - Erosive esophagitis, n (\%) & $7(35)$ & $12(38.7)$ & 0.789 \\
\hline Adverse events, n (\%) & $6(30)$ & $4(12)$ & 0.163 \\
\hline Length of hospital stay; mean \pm SD & $3.75 \pm 1.45$ & $3.58 \pm 0.99$ & 0.622 \\
\hline
\end{tabular}

the Chagasic group, two (10\%) patients had prior treatment for achalasia; one (5\%) patient had a prior pneumatic dilation and one (5\%) had LHM. In the idiopathic group, five patients (16\%) had prior treatment for achalasia; two (6\%) had a prior pneumatic dilation, and three (10\%) had LHM. Unfortunately, the two patients in the Idiopathic group whom had undergone previous pneumatic dilation presented with a recurrence of symptoms within the 1-year follow-up period. Wu et al. [24] showed that prior endoscopic or surgical interventions caused submucosal fibrosis, which changed the normal esophageal physiology, increased the difficulty, and affected the quality of POEM surgery. Similarly, Ngamruengphong et al. [25] reported that prior treatment with pneumatic dilation was associated with treatment failure (OR,3.41; $95 \% \mathrm{Cl}, 1.25-9.23)$. Thus, our treatment failure in these individuals may be consistent with the results seen from prior groups and may have been related to their prior therapy for achalasia.

Martinek et al. [26] showed decrease in the Eckardt score (7.0 vs. $0.0, P<0.05)$ and increase in mean BMI (25.3 vs. 25.9, $P>0.05)$ at a follow-up of 1 year, and decrease in LESP $(41.7+$ 20.1 vs. $21.3+11.5, P<0.05)$ at a follow-up of 3 months at high-resolution manometry. Ngamruengphong et al. [25] showed decrease in the Eckardt score (6.6 vs. $0.8, p<0.05$ ) at a follow-up of 1 year. Barbieri et al. [23] showed a median time for POEM of 156 minutes (range: 42-112), with a length of hospital stay of 3.65 days $(+1.18)$, and a median myotomy length of $10 \mathrm{~cm}$ (range: $6-14$ ). In our study, we similarly demonstrated a statistical difference in both groups regarding the decrease in the Eckardt score (7.50 vs. 1.00 in the Chagasic group, $P<0.001 ; 8.00$ vs 0.00 in the Idiopathic group, $P<$ $0.001)$ ), LESP (21.76 vs. 10.32 in the Chagasic group, $P<0.001$; 25.21 vs. 10.70 in the idiopathic group, $P<0.001)$, and increase in mean BMI (25.41 vs. 27.11 in the Chagasic group, $P<0.001$; 22.17 vs. 25.99 in the idiopathic group, $P<0.001)$ after the POEM procedure compared to baseline.

While POEM is considered to generally be safe and effective, there are associated AEs that require careful technique and close monitoring of patients both during the case and post-procedurally. AEs that may be encountered during POEM include insufflation-related events, such as capnoperitoneum or pneu- moperitoneum, mucosal injuries, bleeding, or anesthesia related complications. Postprocedurally, AEs that may be encountered include pain or delayed leaks or perforations. Unfortunately, there is no standardized classification system for grading the severity of AEs associated with POEM, resulting in wide variation in their reported occurrences [27]. In our study, AEs occurred in 10 patients (19\%); four esophageal mucosal injuries, five gastric mucosal injuries and one PE. We had double the rate of mucosal injury in the Chagas group ( $25 \%$ vs. $12 \%$ ). We believe that there is a tendency towards a higher rate of mucosal lesions during POEM in patients with chagasic achalasia due to the higher degree of underlying fibrosis. However, in our study, there was no statistical difference despite this trend. In accordance with the ASGE lexicon severity grading system, all AEs were graded as mild [16].

Kahaleh et al. [11] showed that Chagas disease patients with achalasia were 9.5 times more likely to respond to POEM treatment (odds ratio, 9.5; $P<0.020$ ) compared with achalasia patients without the disease. However, in this study, [11] there was a high rate of AEs (34/89) and failure to perform POEM in eight cases (8/89). The main AEs were bleeding (14\%) and pneumoperitoneum (6\%) [11]. In our study, AEs were defined as any symptomatic event requiring temporary stop of the procedure and/or further intervention, any event that prevented completion of the procedure, and/or any event that resulted in prolongation of hospital stay, required another procedure, or subsequent medical consultation. Therefore, we did not consider bleeding with spontaneous resolution to be an AE. In our study, all POEM procedures were performed with a full-thickness myotomy technique, with a 3 - to $4-\mathrm{cm}$ myotomy below the cardia, making pneumoperitoneum an expected event. Thus, for this reason, we did not consider pneumoperitoneum requiring decompression to be an $\mathrm{AE}$.

This study has some limitations. First, the retrospective design has the inherent limitations associated with all studies that are retrospective in nature. Second, all patients in our center were evaluated by conventional rather than high-resolution manometry. High-resolution manometry is the gold standard for evaluation of esophageal motor disorders, but conventional manometry remains an excellent diagnostic tool [28]. Due to 
the high costs for performing high-resolution manometry and low availability in our center, we chose to perform only conventional manometry for pre- and post-POEM evaluation. And third, our follow-up period is limited to 1 year, which limits our ability to report the long-term results of POEM in these patient groups.

\section{Conclusion}

POEM is an effective and safe treatment for patients with achalasia, with no difference in outcomes comparing Chagasic and idiopathic etiology.

\section{Competing interests}

The authors declare that they have no conflict of interest.

\section{References}

[1] Vaezi MF, Pandolfino JE, Vela MF. ACG clinical guideline: diagnosis and management of achalasia. Am J Gastroenterol 2013; 108: 1238-1249

[2] Yu L, Li ], Wang T et al. Functional analysis of long-term outcome after Heller's myotomy for achalasia. Dis Esophagus 2010; 23: 277-283

[3] Reynolds JC, Parkman HP. Achalasia. Gastroenterol Clin North Am 1989; 18: 223

[4] de Oliveira RB, Rezende Filho J, Dantas RO et al. The spectrum of esophageal motor disorders in Chagas' disease. Am J Gastroenterol 1995; 90: 1119-1124

[5] Mayberry JF. Epidemiology and demographics of achalasia. Gastrointest Endosc Clin N Am 2001; 11: 235-248

[6] Winter H, Shukla R, Elshaer M et al. Current management of achalasia - a review. Br J Med Pract 2015; 8: a810

[7] Eckardt DF. Clinical presentations and complications of achalasia. Gastrointest Endosc Clin N Am 2001; 11: 281-292

[8] Inoue H, Minami H, Kobayashi Y et al. Peroral endoscopic myotomy (POEM) for esophageal achalasia. Endoscopy 2010; 42: 265-271

[9] Ramchandani M, Nageshwar Reddy D, Nabi Z et al. Management of achalasia cardia: Expert consensus statements. J Gastroenterol Hepatol 2018; 33: 1436-1444

[10] Bonifácio P, de Moura DTH, Bernardo WM et al. Pneumatic dilation versus laparoscopic Heller's myotomy in the treatment of achalasia: systematic review and meta-analysis based on randomized controlled trials. Dis Esophagus 2019: 32
[11] Kahaleh M, Xu MM, Zamarripa F et al. POEM in Latin America: the rise of a new standard. J Clin Gastroenterol 2019; 53: e352-e355

[12] Rezende JM. Classificação radiológica do megaesôfago. Res Goiana Med 1982; 28: 187-191

[13] Eckardt VF, Aignherr C, Bernhard G. Predictors of outcome in patients with achalasia treated by pneumatic dilatation. Gastroenterol 1992; 103: $1732-1738$

[14] Lundell LR, Dent J, Bennett JR et al. Endoscopic assessment of oesophagitis: clinical and functional correlates and further validation of the Los Angeles classification. Gut 1999; 45: 172-180

[15] Cotton PB, Eisen GM, Aabakken L et al. A lexicon for endoscopic adverse events: report of an ASGE workshop. Gastrointest Endosc 2010; 71: 446-454

[16] Rosner B. Fundamentals of Biostatistics.2 Boston: PWS Publishers; 1986: $584-585$

[17] Timm NH. Multivariate Analysis with Applications in Education and Psychology. Monterrey, CA: Brooks/Cole; 1975: 687-688

[18] Boeckxstaens GE. Achalasia: virus-induced euthanasia of neurons? Am J Gastroenterol 2008; 103: 1610-1612

[19] Troncon LE, Oliveira RB, Romanello LM et al. Abnormal progression of a liquid meal through the stomach and small intestine in patients with Chagas' disease. Dig Dis Sci 1993; 38: 1511-1517

[20] Spiess AE, Kahrilas PJ. Treating achalasia: from whalebone to laparoscope. JAMA 1998; 280: 638-642

[21] Boeckxstaens GE, Zaninotto G, Richter JE. Achalasia. Lancet 2014; 383: 83-93

[22] Zaninotto G, Bennett C, Boeckxstaens G et al. The 2018 ISDE achalasia guidelines. Dis Esophagus 2018; 31: 1-29

[23] Barbieri LA, Hassan C, Rosati R et al. Systematic review and meta-analysis: Efficacy and safety of POEM for achalasia. United European Gastroenterol J 2015; 3: 325-334

[24] Wu QN, Xu XY, Zhang XC et al. Submucosal fibrosis in achalasia patients is a rare cause of aborted peroral endoscopic myotomy procedures. Endoscopy 2017; 49: 736-744

[25] Ngamruengphong S, Inoue $\mathrm{H}$, Chiu PW et al. Long-term outcomes of per-oral endoscopic myotomy in patients with achalasia with a minimum follow-up of 2 years: an international multicenter study. Gastrointest Endosc 2017; 85: 927-933

[26] Martinek ], Svecova H, Vackova Z et al. Per-oral endoscopic myotomy (POEM): mid-term efficacy and safety. Surg Endosc 2018; 32: $1293-$ 1302

[27] Nabi Z, Reddy DN, Ramchandani M. Adverse events during and after per-oral endoscopic myotomy: prevention, diagnosis, and management. Gastrointest Endosc 2018; 87: 4-17

[28] Gao Q, Tu S, Tang X et al. Mid-term outcomes of a single center randomized controlled trial comparing peroral endoscopic short versus long myotomy for achalasia in China. Am J Gastroenterol 2017; 112: S173-S174 